\title{
Illegal parking road recognition based on video detection equipment
}

\author{
Xinyue Fan ${ }^{1,2, a}$, Qi Shen ${ }^{3}$, Qinglong $\mathrm{He}^{2}$ \\ ${ }^{1}$ Guizhou University Guizhou Provincial Key Laboratory of Public Big Data, Guizhou Guiyang, 550025,China \\ ${ }^{2}$ Guizhou University, School of Mathematics and Statistics, Guizhou Guiyang, 550025, China \\ ${ }^{3}$ Guiyang Public Security Traffic Administration Bureau, Science and Technology Department,Guizhou Guiyang,550081,China
}

\begin{abstract}
Based on the big data collected by the video detection equipment, the network topology table of the city level video detection equipment is constructed by using the time relation and the spatial position relation of the data. By using the steepest descent method and adaptive method, the travel confidence time randomness model is constructed, which can describe whether a traveler can finish his travel time on time. It overcomes the shortcomings of the existing travel time reliability calculation model, which is difficult to combine with the actual use of video detection equipment data, then examples analysis are followed. The results show that, for the data collected by the video detection device, the travel confidence time randomness model is more accurate than the existing models. It can describe the probability of the traveler arriving at the destination in a given time more accurately, which can be used to identify illegal parking road and provide a reliable basis for traffic management departments in traffic planning, dividing road network status and traffic situation prediction.
\end{abstract}

\section{Introduction}

Travel confidence time refers to the confidence interval of a vehicle passing through two test sections under a given confidence level under the condition of not parking for a long time. It is similar to the reliability of travel time to describe whether a traveler can complete his trip on time. The difference between the travel time reliability and the travel confidence time is that the travel confidence time not only gives the upper limit of the interval of the reliable travel time, but also gives the lower limit, which can more accurately describe the traveler's probability of arriving at the destination within a given time. The travel confidence time is not only valuable in the illegal parking road recognition and traffic congestion identification, but also in the analysis of deck cars, the accuracy of license plate recognition and the dissipation rate of road network vehicles.

In recent years, with the emergence of star-level and black-light-level video detection equipment, the accuracy of the data collected by video detection equipment is getting higher and higher, and the types of data are becoming richer and richer. Compared with other traffic data detection equipments, video detection equipment has a high cost-effective performance, coupled with the demand for illegal data acquisition, and video detection equipment has been installed in many cities by large scale in China. Video detection equipment can obtain a lot of information of vehicle such as license plate, vehicle type, passing time, lane number, direction, and so on. Then it can be obtained that traffic flow, single-car travel time, section travel time and other information, in which section travel time is more objective and accurate than using GPS information to get. Many estimation and prediction researches for travel time are based on video detectors ${ }^{[1-4]}$.

Kazagli $^{[5]}$ estimates the travel time of urban trunk roads by using the data of vehicle identification system; Yang et al. ${ }^{[6]}$ proposes a practical travel time prediction algorithm based on the real-time travel time data collected by the license plate automatic recognition system and analyzes the error of the prediction results; Zhang ${ }^{[7]}$ established a link travel time estimation model based on the spatial Markov chain; According to the relationship between link travel time estimation parameters and sample rate, and the spatio-temporal variation characteristics and significance analysis results of actual travel time matching rate, $\mathrm{Fu}$ et al. ${ }^{[2]}$ determined the feasibility of estimating travel time with video detection equipment data; $\mathrm{Li}^{[8]}$ established a calculation model of vehicle travel time in road section and traffic delay model in downstream signalized road intersection. Liu et al. ${ }^{[9,10]}$ extended the model to estimate the travel time of trunk road in real time under congestion condition by tracking the trajectory and braking of virtual detectors. These models are based on the accuracy of the detection equipment is higher than $98 \%$, in fact, the equipment can not be put into use to achieve such a high precision. Daganzo ${ }^{[11]}$ insists that the traffic network under saturated conditions has complex characteristics, and there are unpredictable traffic game behaviors among travelers on the road network, which

${ }^{a}$ Corresponding author: fan.xinyue $@ 163 . c o m$ 
makes it difficult to study the travel time reliability of the road network based on traffic assignment theory. At present, almost all of the above-mentioned researches are based on static OD analysis to get the travel time and its probability distribution, and then to estimate the reliability. These models basically make very strong theoretical assumptions, such as travel time obeys normal distribution, Weill distribution, exponential distribution and so on ${ }^{[12]}$. These assumptions have a decisive impact on the reliability of the final travel time and are difficult to apply to the actual traffic network. Zhang ${ }^{[13]}$ and others realized the real-time estimation of urban road network reliability from the real traffic flow and signal timing data of each intersection of the road network. Unfortunately, the data used is the flow data collected by the coil detector, and does not contain the key data such as the license plate and the passing time.

The data used in this paper are all from the $80 \mathrm{~PB}$ data of all the video detection equipments in Guiyang. According to the spatial-temporal correlation, the network topology of the video detection equipment is established by using the original traffic data, and the confidence time stochastic model of the section travel is constructed by the steepest descent method and selfadaptively. The stochastic model is applied to the detection section of Guiyang road network with the relationship between upstream and downstream, and it used to recognize the illegal parking road section. The model has applicability in other areas, and it can be widely applied after necessary verification.

\section{Network topology of video detection device}

Network topology of video detection equipment is used to describe the structure relationship between video detection equipment in the road network, such as which video detection equipment is in the same section, which equipment exists upstream and downstream relationship, physical distance between equipment and so on. In order to reflect the generality and practicability of the model, it is necessary to analyze the travel confidence time of each pair of cross-section with upstream and downstream relationship based on the network topology of video detection equipment. However, it is difficult for traffic management departments in many cities to give a completely accurate network topology table of video detection equipment. There are three main reasons: firstly, because many urban traffic video detection systems are divided into several phases of construction, may be different from each phase of the construction unit, the basic information of the equipment is not summarized; secondly, because the video detection equipment itself does not install GPS module, the data obtained by GPS acquisition equipment and the actual installation position deviation is very large. Third, because of the complex road network structure, equipment installation density is small, not all equipment are enclosed region, equipment upstream and downstream relationship is difficult to fully clarify. Therefore, it is necessary to use the actual data to establish the network topology table of video detection equipment to clarify the structure relationship between devices. In addition, the network topology table of video detection equipment needs to be updated periodically and automatically because of the new equipment and relocation. In this regard, the following steps are given to build a video to detect the topology of road devices:

Step 1: Collect all the original data of the road network, extract the time that the same license plate was detected by different video detection equipment within a month, then construct the vehicle trajectory according to the time sequence that the vehicle was identified, and transform it into the device network topology table. The main field records the upstream equipment flow data, the downstream equipment flow data and the track number and direction of the section.

Step 2: In the network topology table constructed by Step 1, a statistic is constructed based on the traffic data detected by the upstream and downstream video detection devices in this direction as follows:

$$
\mu=\frac{N_{O D}}{\min \left(N_{S}, N_{A}\right)}
$$

Here $N_{S}$ is the traffic flow at this section intersection in one month, $N_{A}$ is the upstream traffic flow, $N_{O D}$ is the matching traffic flow of two section license plates.

Step 3: The statistics of each road section in the topology table of equipment network are classified, and the outliers of different road sections are detected by box diagram. Clean the topology of the network topology.

Step 4: Comparing the equipment network topology table with the GPS data given by the construction unit, point calibration and on-site manual comparison are carried out on Google map. Combined with the spatial location of equipment, the topology table of equipment network is optimized. Some results are shown in Table 1.

Table.1 Network topology of some video detection devices in Guiyang

\begin{tabular}{|c|c|c|c|c|}
\hline Source & Direction & Taget & Direction & trajectories \\
\hline Baoshan North Road (Xinyin chang) & 3 & Guanshan East Road (Da Guan) & 1 & 87606 \\
\hline Baoshan North Road (Xinyin chang) & 3 & Xintian \& Chemical Road & 3 & 148935 \\
\hline Baoshan North Road (Xinyin chang) & 3 & Beijing road \& Baoshan North Road & 3 & 133307 \\
\hline Baoshan North Road (Xinyin chang) & 3 & Baoshan Road & 4 & 283013 \\
\hline Baoshan North Road (Xinyin chang) & 4 & Baoshan North road \& Yanan East Road & 4 & 324542 \\
\hline Baoshan North Road (Xinyin chang) & 4 & Youyi Road (tobacco companies) & 4 & 294502 \\
\hline Baoshan North Road \& Dongxin Road & 3 & Wenchang North Road \& Dongxin Road & 1 & 18636 \\
\hline Baoshan North Road \& Dongxin Road & 3 & Baoshan North Road \& Xudong Road & 3 & 215977 \\
\hline Baoshan North Road \& Dongxin Road & 4 & Wenchang North Road \& Dongxin Road & 1 & 75335 \\
\hline Baoshan North Road \& Dongxin Road & 4 & Baoshan North Road \& Xudong Road & 3 & 15215 \\
\hline Baoshan North Road \& Dongxin Road & 4 & Baoshan South Road \& Zhongshan East & 4 & 57951 \\
\hline
\end{tabular}




\begin{tabular}{|c|c|c|c|c|}
\hline Baoshan North Road \& Xudong Road & 1 & Baoshan North Road \& Yanan East Road & 3 & 17852 \\
\hline Baoshan North Road \& Xudong Road & 1 & Baoshan North Road \& Dongxin Road & 4 & 30280 \\
\hline$\ldots$ & $\ldots$ & $\ldots$ & $\cdots$ & $\ldots$ \\
\hline
\end{tabular}

\section{Travel confidence time}

\subsection{Vehicle actual travel time}

By calculating the travel time of the same vehicle, the difference between the passing time collected by the downstream equipment and the upstream equipment is often used. However, some special circumstances easily lead to excessive time delay of individual vehicles, such as midway anchorage, parking lot stay and so on. In addition, if the equipment exists such as inaccurate calibration, power failure, missed detection or mistake detection, it may lead to the same license plate obtained from the two sections of the second trip, or even multiple trips. The next day trip, at this time the running state of individual vehicles can not accurately reflect the operation of traffic. In fact, there is no uniform and reliable standard for how to identify these special cases and how to select the threshold. Next, the actual travel time of a vehicle is obtained from the actual road network passing data to seek thresholds for identifying such special cases.

Let the time information of passing data collected by video detection equipment is taken as sample space, the data are randomly sampled and divided into mid-day period (peak period: 07:00-09:00, 17:30-19:30, ordinary period: 9:00-17:30, 19:30-00:00, low peak period: 0007:00) according to the morning and evening peaks and peacetime. The actual travel time difference between the upstream and downstream sections of the same vehicle in one day is taken as a random variable $\theta$. Figure 1 is the channelization map of Baoshan North Road in Guiyang. The intersection of Baoshan North Road and Xudong Road $S$ taken as the experimental intersection. The upstream A is the intersection of Baoshan North Road and Dongxin District Road (the intersection of provincial Public Security Department), and the downstream B is the intersection of Baoshan North Road and Yan'an East Road (the intersection of Normal University).

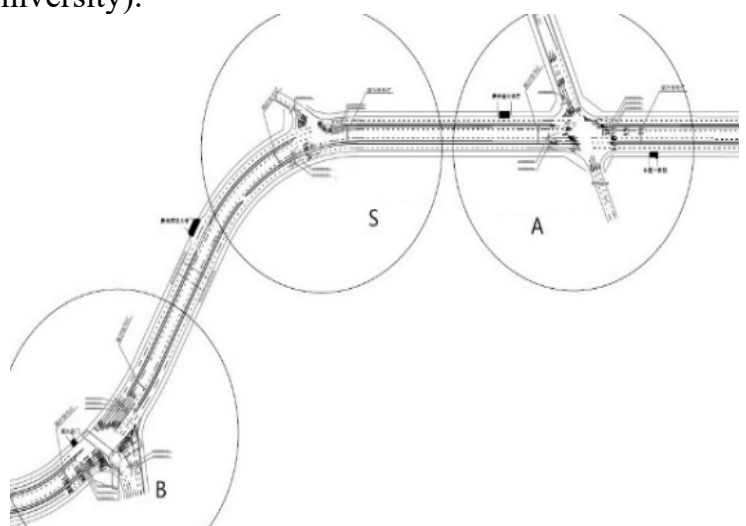

Figure 1. Map of Baoshan North Road in Guiyang

Vehicles identified by video detection equipment from $\mathrm{S}$ to $\mathrm{B}$ are matched by 315509588 passing data in July 2017 and extracted according to the shortest actual travel time (if two devices control a lane at the same time, the first capture time of the same section is taken). Statistics of the actual travel time of vehicles take $5 \mathrm{~s}$ as a frequency, intercepting a single day through the actual travel time of the road section is called truncation time. The maximum cut-off time of the experimental section is 2 hours, or $7200 \mathrm{~s}$. Frequency histogram of the peak and low peak periods of the section is shown in Figure 2. The abscissa is the actual travel time of the section and the ordinate is the passing frequency. According to the large sample theory, the sum of independent and identically distributed random variables can be regarded as normal variables. Theoretically, for random variables, the normalized approximate normal distribution, according to the Laida criterion, the confidence can reach $99.74 \%$. Unfortunately, the actual results can not reach such an ideal situation.

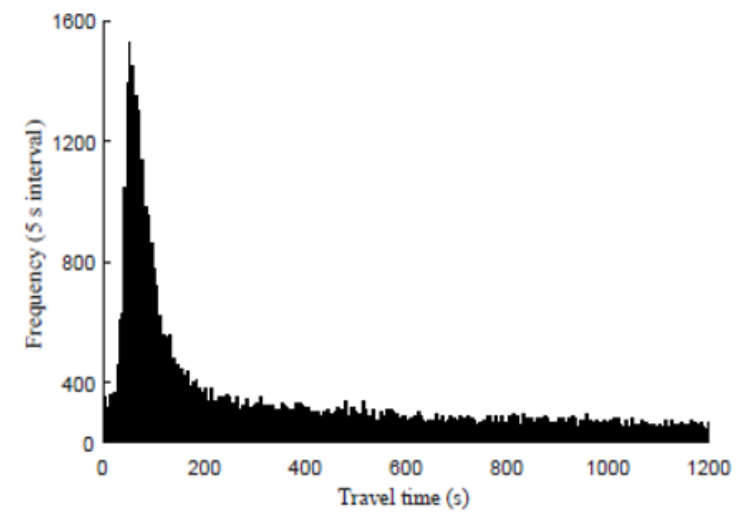

(a) Peak period

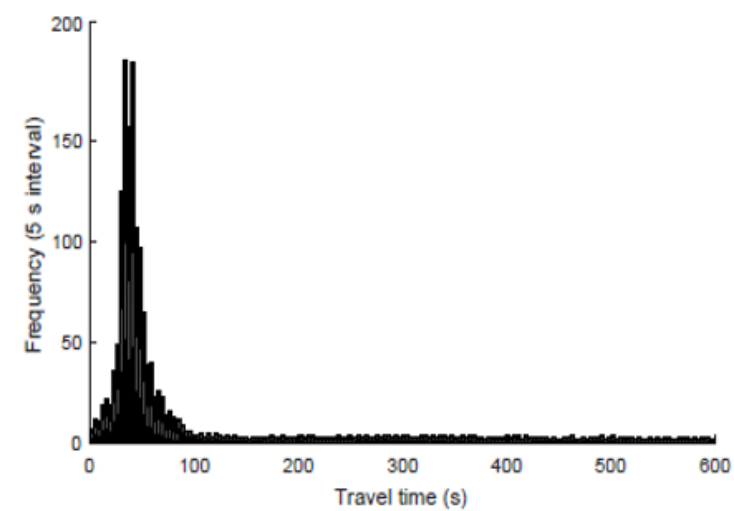

(b) Low peak period

Figure 2. Frequency histogram of intersection of Baoshan north and Xudong road to Baoshan north road and Yanan East road

As can be seen from Figure 2 that the night data is better, if the cut-off time is shortened to 200 seconds in the low peak period, it can be tested by normal distribution K-S test. If the truncation time is changed during the peak period, the actual data still can not pass the normal distribution K-S test, and do not obey some statistical distribution characteristics. Therefore, it is unreasonable to assume that the travel time obeys normal distribution or other distribution. Traffic flow state is random, time variant and has long tail phenomenon ${ }^{[14]}$. 


\subsection{Section travel confidence time}

Based on the license plate recognition data, the estimation methods of the single-car travel speed and the average interval travel speed are designed in paper [15], but the threshold calculation of the traffic congestion recognition speed mentioned in the paper is difficult to obtain accurately in practice. In the Netherlands National Transport Policy Document Nota Mobility, the time threshold for travel less than 50 kilometers is 1.2 times the average travel time, which is considered reliable within the threshold range, otherwise unreliable. This standard is widely applied to the reliability judgement of travel time in domestic transportation field. The policy objective is that 90 times of 100 trips are reliable, that is, $90 \%$ confidence level can be achieved. Whether the reliability of the actual travel time of the road section can be followed by this standard remains to be verified. The results of the actual travel time will be given in the fourth part of this paper. This standard is not applicable based on the complex traffic flow conditions. In this paper, the travel confidence time $\left[\theta_{L}, \theta_{R}\right]$ based on the actual application is proposed.

Definition. Let $\theta$ be parameter of total travel time, here parameter space is $\Theta$, Actual travel time are samples $\mathrm{t}_{1}, \mathrm{t}_{2}, \mathrm{t}_{3}, \ldots, \mathrm{t}_{n}$ which come from the general population. For fix $\alpha(0<\alpha<1)$, Suppose there are two statistics $\theta_{L}=\theta_{L}\left(t_{1}, t_{2}, \ldots, t_{n}\right)$ and $\theta_{R}=\theta_{R}\left(t_{1}, t_{2}, \ldots, t_{n}\right)$, for any $\theta \in \Theta$,

$$
P\left\{\theta_{L} \leq \theta \leq \theta_{R}\right\} \geq 1-\alpha
$$

Then the interval $\left[\theta_{L}, \theta_{R}\right]$ is called $\theta$ travel confidence time with a confidence level of $1-\alpha \cdot \theta_{L}$ is lower confidence limit, and $\theta_{R}$ is upper confidence limit.

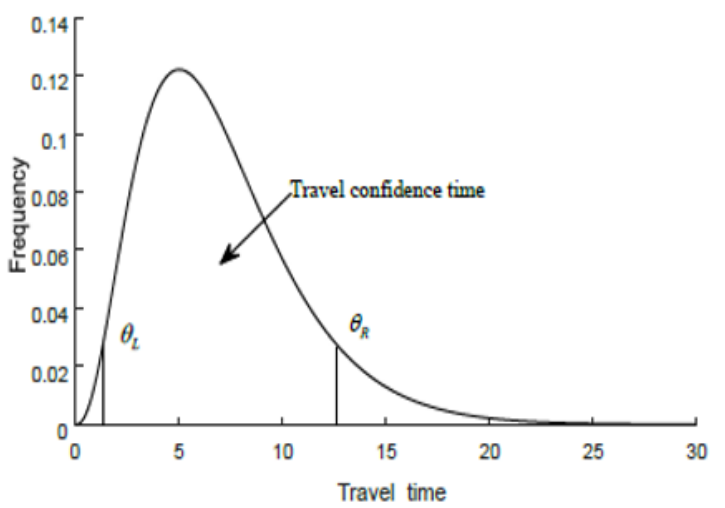

Figure 3. Travel Confidence Time

Section travel confidence time gives a threshold based on actual travel time reliability. Compared with travel time reliability, it not only gives the lower limit of travel time, but also gives the upper limit of travel time (see Figure 3), so it can more accurately describe section travel time. There are several key factors that affect the confidence time of travel: confidence, truncation time and convergence conditions. Next, we discuss the modeling process of the confidence time randomness model of link travel.

Take the intersection of Baoshan North Road and Xudong Road S to Baoshan North Road and Yan'an East Road intersection B as the experimental object as in Section 3.1. Firstly, the maximum frequency is selected as the initial frequency from the frequency data, and the corresponding 5 seconds are taken as the initial travel confidence time. Left and right extensions were calculated for the first $5 s$ and the next $5 s$ frequencies. High frequency of passing was selected as the extension of travel confidence time. Extrapolation of travel confidence time interval (such as initial time interval $[20 s, 25 s]$, the next $5 s$ frequencies higher than the previous $5 s$ frequencies, and the travel confidence time interval was expanded to $[20 s, 30 s]$. By analogy, until $95 \%$ of the vehicles pass within the travel confidence time, that is, 95\% confidence degree. Because the tail phenomenon does not disappear in actual road network, the travel confidence time depends on the truncation time. The truncation time is extended to the right in 300 seconds after the initial value of truncation time is 600 seconds, and the confidence time with $95 \%$ confidence degree is obtained at the significance level of 0.05 . It is found that the travel confidence time does not change any more and converges to a stable interval (see Table 2). The peak period is stable as $[30 s, 225 s]$, the low peak period is stable as $[10 \mathrm{~s}, 160 \mathrm{~s}]$, and the actual travel time upper and lower limits of the low peak period are lower than the peak period.

In the road network, some sections with better peak conditions are selected as the experimental objects. The middle section of the road without fork roads, residential areas and large parking lot can be regarded as a closed area. For example, Zhonghua Road \& provincial road intersection to the fountain section, the section is mainly controlled by traffic lights, there will be no congestion, the actual travel time is concentrated, no trailing phenomenon. Another section of Beijing west road \& the intersection of Zhongba road to Jiaxiu south road Wulichong section has trailing, trailing value is small, the data quality is good (see Figure 4,5). The initial value of truncation time is $1800 \mathrm{~s}$, and the significance level is 0.05 . Table 3 shows that the confidence time of the two sections will converge to a stable interval and converge quickly.

Table 2 Travel Confidence Time of intersection of Baoshan North Road \& Xudong Road to intersection of Baoshan North Road \& Yanan East Road

\begin{tabular}{|c|c|c|c|}
\hline \multirow{2}{*}{ Truncation time $(s)$} & \multicolumn{2}{|c|}{ Peak period } & Low peak period \\
\cline { 2 - 4 } & Confidence degree $(\%)$ & {$\left[\theta_{\mathrm{L}}, \theta_{R}\right]$} & {$\left[\theta_{\mathrm{L}}, \theta_{R}\right]$} \\
\hline 600 & 0.95 & {$[30 \mathrm{~s}, 190 \mathrm{~s}]$} & {$[25 \mathrm{~s}, 145 \mathrm{~s}]$} \\
\hline 900 & 0.95 & {$[30 \mathrm{~s}, 210 \mathrm{~s}]$} & {$[20 \mathrm{~s}, 150 \mathrm{~s}]$} \\
\hline 1200 & 0.95 & {$[30 \mathrm{~s}, 215 \mathrm{~s}]$} & {$[20 \mathrm{~s}, 155 \mathrm{~s}]$} \\
\hline 1500 & 0.95 & {$[30 \mathrm{~s}, 220 \mathrm{~s}]$} & {$[15 \mathrm{~s}, 155 \mathrm{~s}]$} \\
\hline 1800 & 0.95 & {$[30 \mathrm{~s}, 225 \mathrm{~s}]$} & {$[10 \mathrm{~s}, 160 \mathrm{~s}]$} \\
\hline 2100 & 0.95 & {$[30 \mathrm{~s}, 225 \mathrm{~s}]$} & {$[10 \mathrm{~s}, 160 \mathrm{~s}]$} \\
\hline
\end{tabular}


Table 3 Travel Confidence Time of Zhonghua road \& provincial road intersection to the fountain \& Beijing west road \& the intersection of Zhongba to Jiaxiu south road Wulichong

\begin{tabular}{|c|c|c|c|c|}
\hline \multirow{2}{*}{ Truncation time (s) } & $\begin{array}{c}\text { Zhonghua road \& provincial road intersection to the } \\
\text { fountain \& Beijing west road }\end{array}$ & \multicolumn{2}{c|}{$\begin{array}{c}\text { Beijing west \& the intersection of Zhongba to } \\
\text { Jiaxiu south road Wulichong }\end{array}$} \\
\cline { 2 - 5 } & Confidence degree (\%) & {$\left[\theta_{\mathrm{L}}, \theta_{R}\right]$} & Confidence degree (\%) & {$\left[\theta_{\mathrm{L}}, \theta_{R}\right]$} \\
\hline 1800 & 0.95 & {$[5 \mathrm{~s}, 95 \mathrm{~s}]$} & 0.95 & {$[235 \mathrm{~s}, 585 \mathrm{~s}]$} \\
\hline 1740 & 0.95 & {$[5 \mathrm{~s}, 95 \mathrm{~s}]$} & 0.95 & {$[235 \mathrm{~s}, 585 \mathrm{~s}]$} \\
\hline 1680 & 0.95 & {$[5 \mathrm{~s}, 95 \mathrm{~s}]$} & 0.95 & {$[235 \mathrm{~s}, 585 \mathrm{~s}]$} \\
\hline
\end{tabular}

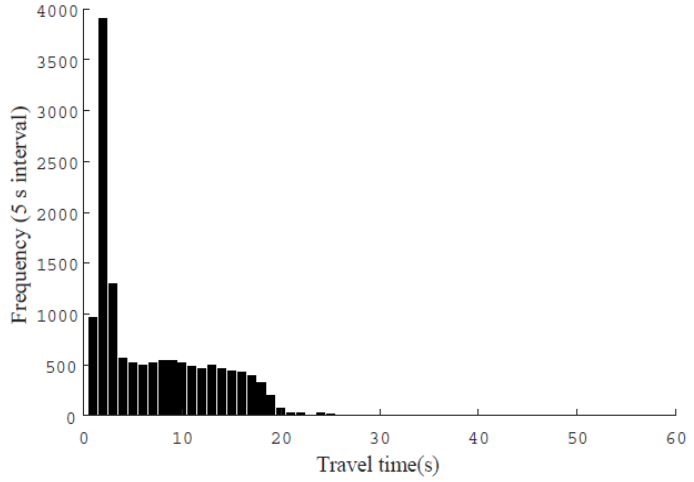

Figure 4 Frequency histogram of Zhonghua Road and Provincial road intersection to the fountain

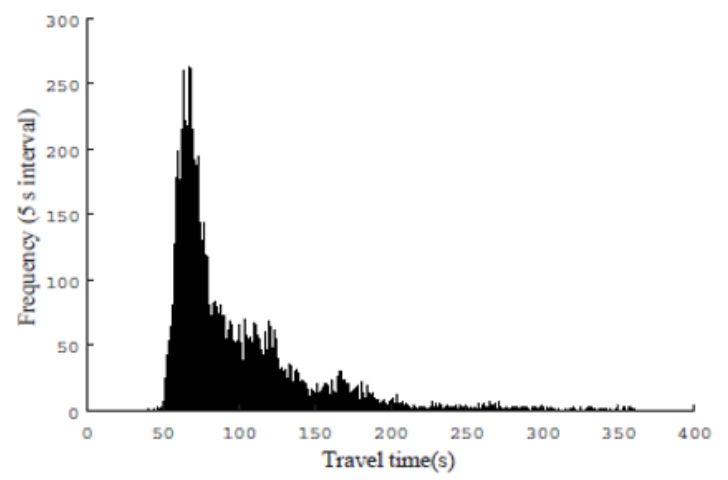

Figure 5 Beijing west road and the intersection of Zhongba to Jiaxiu south road and Wulichong

For some congested roads, there may be bimodal distribution $^{[16]}$. The confidence time is slow or even convergent. Two easy congested roads are chosen as the experimental objects. Because the trailing part of the data value is larger (Figure 6,7), that is, because there are intersections on the road, residential areas, parking lots and so on, a large number of vehicles stay too long. The significance level is 0.05 , and the confidence level is $95 \%$. If we still take a high degree of confidence, then the actual long-stay vehicles will affect the description of travel time, but also affect the reliability of travel time is an unstable factor.

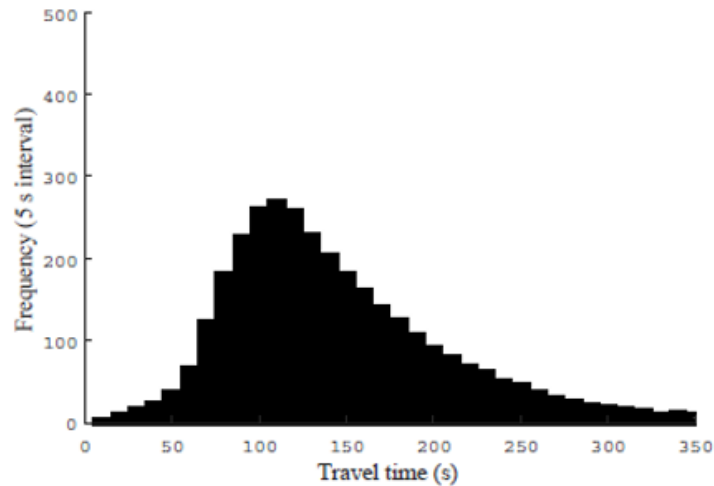

Figure 6 Travel Confidence Time of Jiaxiu south road \& Wulichong to Beijing west Zhongba

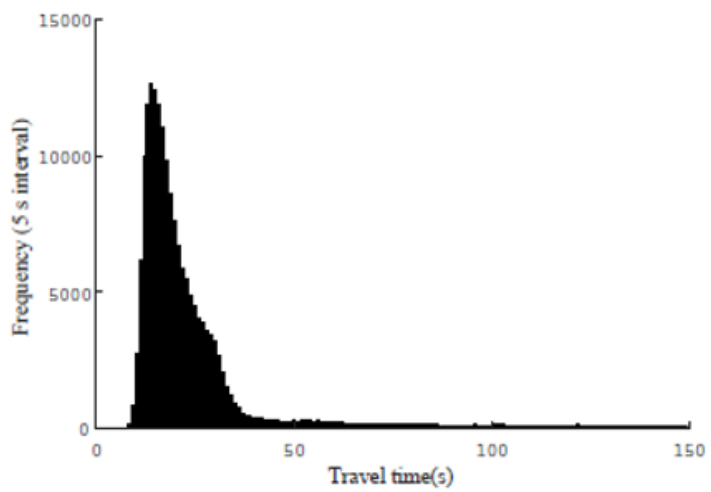

Figure 7 Travel Confidence Time of Baoshan North \& Yanan road intersection to Baoshan \& Xinyinchang

Therefore, truncation time and confidence adaptive method should be adopted to optimize the previous section travel confidence time method. The actual travel time $\theta$ collected, samples are $t_{1}, t_{2}, t_{3}, \ldots, t_{n}$, it's distribution function is $F(\theta)=\sum_{t_{i} \leq \theta} P\left(t_{i}\right)$, Frequency interval is $\tau$. The steepest descent method is used to construct the stochastic model of travel time confidence.

Step 1. Taking the confidence level is $\alpha, \alpha_{0}$ is initial value. i.e. confidence degree is $100\left(1-\alpha_{0}\right) \% \cdot S_{0}$ be the initial of truncation time $S$.

Step 2. For parameters $\alpha, S$, Let $P_{0}=\max P(\theta), P_{0}$ corresponds to the initial value of travel time be $\left[\theta_{0}^{L}, \theta_{0}^{R}\right]$.

Step 3. Defining differential operators

$$
\nabla P\left(\theta_{k}^{L}, \theta_{k}^{R}\right)=P\left(\theta_{k}^{R}+\tau\right)-P\left(\theta_{k}^{L}-\tau\right)
$$

$\left[\theta_{k+1}^{L}, \theta_{k+1}^{R}\right]$. The steepest descent method is: 


$$
\begin{aligned}
& \theta_{k+1}^{L}=\theta_{k}^{L}-\tau D_{L}\left(\theta_{k}^{L}, \theta_{k}^{R}\right) \\
& \theta_{k+1}^{R}=\theta_{k}^{R}+\tau D_{R}\left(\theta_{k}^{L}, \theta_{k}^{R}\right)
\end{aligned}
$$

Here

$$
\begin{aligned}
D_{L}\left(\theta_{k}^{L}, \theta_{k}^{R}\right) & = \begin{cases}0, & \nabla P\left(\theta_{k}^{L}, \theta_{k}^{R}\right)>0 \\
1, & \nabla P\left(\theta_{k}^{L}, \theta_{k}^{R}\right) \leq 0\end{cases} \\
D_{R}\left(\theta_{k}^{L}, \theta_{k}^{R}\right) & = \begin{cases}1, & \nabla P\left(\theta_{k}^{L}, \theta_{k}^{R}\right)>0 \\
0, & \nabla P\left(\theta_{k}^{L}, \theta_{k}^{R}\right) \leq 0\end{cases}
\end{aligned}
$$

Iteration termination condition is

$$
P_{\theta}\left\{\theta_{k+1}^{L} \leq \theta \leq \theta_{k+1}^{R}\right\} \geq 1-\alpha
$$

If (7) satisfies, then the output truncation time is $S,\left[\theta_{S}^{L}, \theta_{S}^{R}\right]$ is confidence time under confidence degree $100(1-\alpha) \%$, here $\theta_{S}^{L}=\theta_{k+1}^{L}, \theta_{S}^{R}=\theta_{k+1}^{R}$. If $S=S_{0}$ denote $\hat{\theta}_{S}^{L}=\theta_{S}^{L}, \hat{\theta}_{S}^{R}=\theta_{S}^{R}$.

Step 4. The truncation time adaptive parameter is $v$. $S=S-v$, Execution Step 2-Step3 repeatly, judge whether convergence is allowed, and allow the error to be any positive real number. $\mathcal{E}$, if

$$
\begin{aligned}
& \left|\theta_{S}^{L}-\theta_{S-v}^{L}\right| \leq \varepsilon \\
& \left|\theta_{S}^{R}-\theta_{S-v}^{R}\right| \leq \varepsilon
\end{aligned}
$$

Then execute Step 7. Otherwise $S=S-v$, do Step 2Step3, until $S<=\hat{\theta}_{S}^{R}$, If the convergence condition is not satisfied (8), Step 5 is implemented.

Step 5. $S=S_{0}+v$, Repeat do Step 2-Step3, Judge whether convergence, if

$$
\begin{aligned}
& \left|\theta_{S}^{L}-\theta_{S+v}^{L}\right| \leq \varepsilon \\
& \left|\theta_{S}^{R}-\theta_{S+v}^{R}\right| \leq \varepsilon
\end{aligned}
$$

Table. 4 Travel Confidence Time of Jiaxiu south road and Wulichong to Beijing west Zhongba to Baoshan north and Yanan road intersection to Baoshan and Xinyinchang

\begin{tabular}{|c|c|c|c|c|}
\hline \multirow{2}{*}{$\begin{array}{c}\text { Truncation time } \\
(s)\end{array}$} & \multicolumn{2}{|c|}{$\begin{array}{c}\text { Jiaxiu south road and Wulichong to Beijing west } \\
\text { Zhongba }\end{array}$} & $\begin{array}{c}\text { Baoshan North Road \& Yan'an East Road to } \\
\text { Xinyinchang of Baoshan North Road }\end{array}$ \\
\cline { 2 - 5 } & Confidence degree (\%) & {$\left[\theta_{L}, \theta_{R}\right]$} & Confidence degree (\%) & {$\left[\theta_{L}, \theta_{R}\right]$} \\
\hline 1800 & 0.95 & {$[200 s, 1035 s]$} & 0.95 & {$[40 s, 935 s]$} \\
\hline 1740 & 0.95 & {$[250 s, 1020 s]$} & 0.95 & {$[40 s, 900 s]$} \\
\hline 1680 & 0.95 & {$[250 s, 985 s]$} & 0.95 & {$[40 s, 860 s]$} \\
\hline$\vdots$ & $\vdots$ & $\vdots$ & 0.95 & {$[40 s, 420 s]$} \\
\hline 1020 & 0.95 & {$[260 s, 870 s]$} & 0.95 & {$[40 s, 975 s]$} \\
\hline 1800 & 0.95 & {$[250 s, 1060 s]$} & 0.95 & $\vdots$ \\
\hline 1860 & 0.95 & $\vdots$ & 0.95 & {$[40 s, 1750 s]$} \\
\hline$\vdots$ & $\vdots$ & {$[230 s, 2405 s]$} & 0.90 & {$[40 s, 360 s]$} \\
\hline 3600 & 0.95 & {$[230 s, 860 s]$} & 0.90 & {$[40 s, 345 s]$} \\
\hline 1800 & 0.90 & {$[230 s, 860 s]$} & 0.90 & {$[40 s, 325 s]$} \\
\hline 1740 & 0.90 & & 0.90 & {$[40 s, 180 s]$} \\
\hline 1680 & 0.90 & & 0.90 & {$[40 s, 180 s]$} \\
\hline 960 & & & & $\vdots$ \\
\hline 900 & & & & $\vdots$ \\
\hline
\end{tabular}

\section{Model test}

Based on the confidence time stochastic model of road network trip in Section 3 above, the confidence travel time of road network was established with July 2007 passing data. Random sampling of August 2017 one day video detection equipment detected by the traffic data,
Then do Step 7. Otherwise $S=S+v$, repeat Step 2-Step3, until $S$ is the maximum truncation time 2 hours, If the convergence condition is not satisfied (9), the output result is "no convergence", and Step 6. is implemented.

Step 6. The confidence adaptive parameter is $\omega, \alpha=\alpha-\omega$, if $1-\alpha \geq 0.8$ Then do Step 2-Step 5, otherwise do Step7.

Step 7. Output travel confidence time final result $\theta_{L}=\theta_{S}^{L}, \theta_{R}=\theta_{S}^{R}$.

The above stochastic model of travel confidence time is applied to the topological structure of all road network video detection equipment in Guiyang with upstream and downstream relationship. The lower limit of the peak and the upper limit of the peak are taken as the upper and lower limit of the travel confidence time. The travel confidence time is calculated by using the above stochastic model in peak and low periods. In Table 2, the travel confidence time between Baoshan North Road \& Xudong Road to intersection of Baoshan North Road \& Yanan East Road is $[10 s, 225 s]$. For the section from Wulichong Road of Jiaxiu South Road to the intersection of Beijing West Road and Zhongba Road, the confidence time converges to $[230 s, 860 s]$ when truncation time is 1680 s, confidence degree falls to $90 \%$. For Baoshan north and Yanan road intersection to Baoshan and Xinyinchang confidence degree falls to $90 \%$, the travel confidence time is $[40 s, 180 s]$. Through manual verification, the convergence time of travel confidence time can better match the actual road conditions, as shown in Table 4.
30 sections of Guiyang, the actual travel time model test (see Figure 8). The solid line in the graph represents the confidence interval of the travel confidence time calculated by the model in this paper. The scattered points are the actual travel time of the same vehicle passing through the section detected by the video detection equipment with the relationship between upstream and downstream. The travel confidence time obtained by this model can include the actual travel time 
threshold under the self-adaptive confidence (the lowest confidence degree is not less than $80 \%$ ).

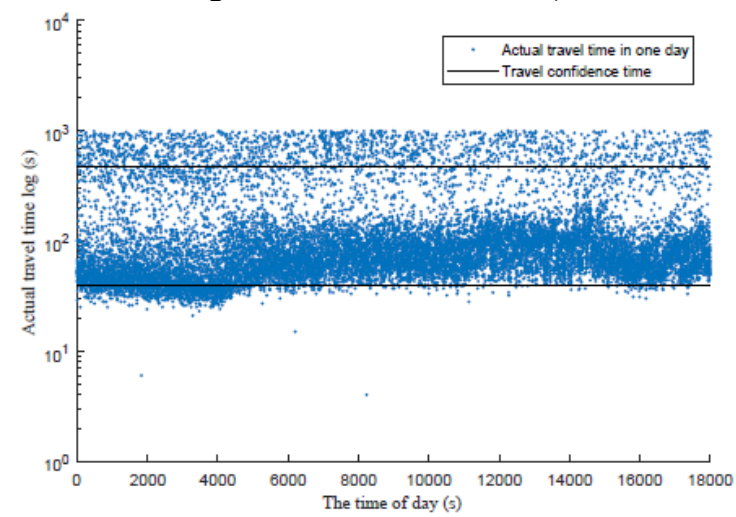

(a)The intersection of Baoshan North Road \& Xudong Road to Baoshan North \& Yanan East Road intersection

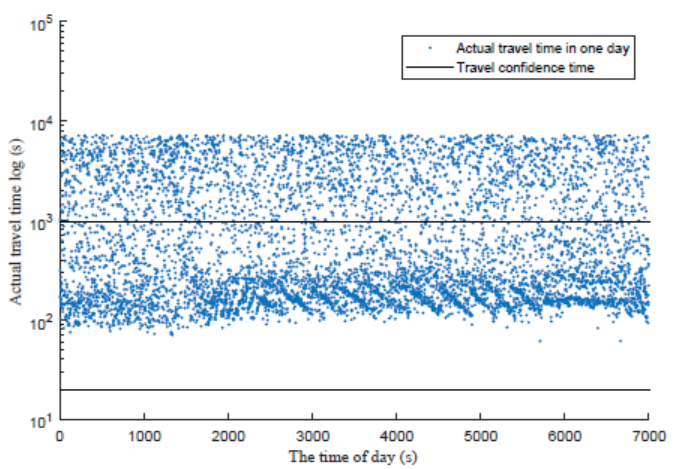

(b)Shachong Road \& Xingguan Road intersection to Zunyi \& Qingyun Road intersection

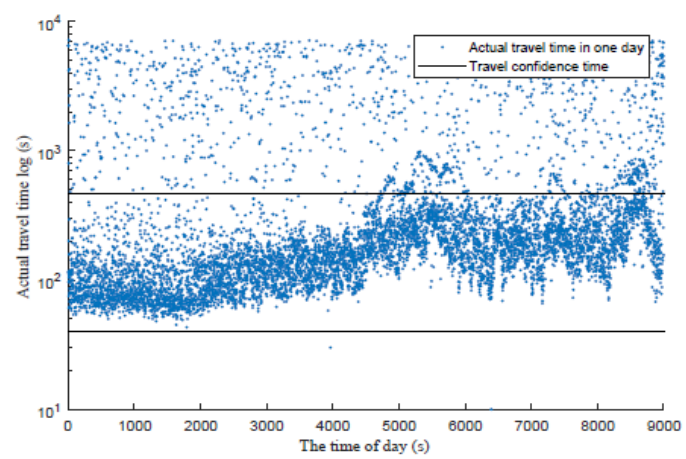

(c)Shachong North Road \& Dagaoqiao intersection to Shachong Road \& Xingguan Road intersection

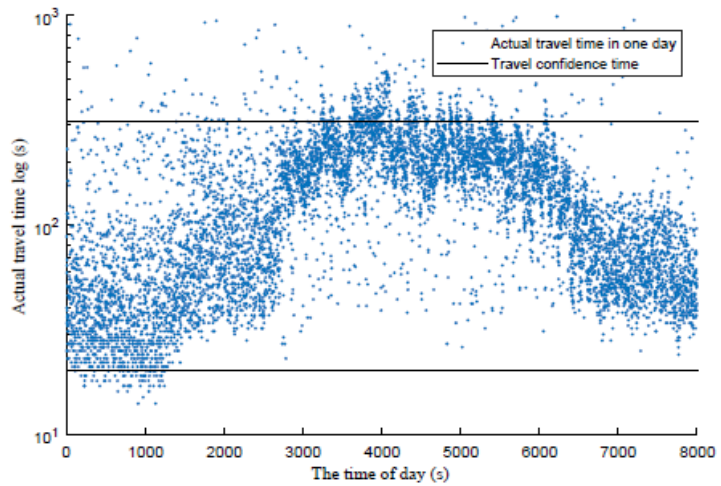

(d)Baoshan South \& Youza Road intersection to Youza Road \& Qingnian Road intersection(right)

Figure 8 The change of the actual travel time.

According to Nota Mobility, the national transport policy document of the Netherlands, the threshold of travel time is 1.2 times the average travel time. Based on this, we have made a necessary verification of the confidence time of road travel defined by us, as shown in Table 5. The travel time reliability given by Holland does not limit the travel time limit. However, there are some zero-crossing times in the actual traffic data of the road network, which can not be used as further data analysis of travel time reliability. As can be seen from the table, their standard range threshold is small. Combined with Figure $8(\mathrm{a})(\mathrm{b})$, the reliability travel time interval given by the Dutch standard are $80 \%$ and $82 \%$ for the two sections, and $76 \%$ and $65 \%$ for the two sections in Figure 8(c)(d), which also contain impossible data sets close to zero. It can not accurately fit the actual travel time, and can not accurately divide the road network condition.

Table.5 Comparison between travel confidence time and Holland travel time reliability standard

\begin{tabular}{|l|c|l|l|l|}
\hline \multicolumn{1}{|c|}{ Upstream intersection } & Downstream intersection & $\begin{array}{l}\text { Confiden } \\
\text { ce degree }\end{array}$ & $\begin{array}{c}\text { Section travel } \\
\text { confidence } \\
\text { time }\end{array}$ & $\begin{array}{c}\text { Holland } \\
\text { standard } 90 \% \\
\text { reliable travel } \\
\text { time }\end{array}$ \\
\hline $\begin{array}{l}\text { Zhonghua Road and Zhonghua } \\
\text { Road }\end{array}$ & Fountain & $95 \%$ & {$[5 s, 95 s]$} & {$[0 s, 96 s]$} \\
\hline Baoshan North and Xudong Road & Baoshan North \& Yanan East & $95 \%$ & {$[10 s, 225 s]$} & {$[0 s, 109 s]$} \\
\hline Wulichong Road Jia Xiu south & $\begin{array}{c}\text { Beijing West Road \& } \\
\text { Zhongba }\end{array}$ & $90 \%$ & {$[185 s, 860 s]$} & {$[0 s, 348 s]$} \\
\hline Beijing West Road \& Zhongba & Wulichong Road & $95 \%$ & {$[205 s, 585 s]$} & {$[0 s, 292 s]$} \\
\hline $\begin{array}{l}\text { Baoshan North \& Yanan East } \\
\text { Road }\end{array}$ & $\begin{array}{c}\text { Baoshan North } \\
\text { Road(Xinying) }\end{array}$ & $90 \%$ & {$[40 s, 180 s]$} & {$[0 s, 223 s]$} \\
\hline $\begin{array}{l}\text { Baihua Mountain \& Daying Road } \\
\text { Read }\end{array}$ & $\begin{array}{c}\text { Beijing and Baoshan North } \\
\text { Road }\end{array}$ & $95 \%$ & {$[10 s, 225 s]$} & {$[0 s, 164 s]$} \\
\hline $\begin{array}{l}\text { Sha Chong Road and Xingguan } \\
\text { Road }\end{array}$ & $\begin{array}{c}\text { Zunyi road and Qingyun } \\
\text { Road }\end{array}$ & $85 \%$ & {$[20 s, 970 s]$} & {$[0 s, 413 s]$} \\
\hline Shachong North Road \& & Sha Chong and Xingguan & $95 \%$ & {$[40 s, 470 s]$} & {$[0 s, 268 s]$} \\
\hline
\end{tabular}




\begin{tabular}{|l|l|l|l|l|}
\hline Dagaoqiao & \multicolumn{1}{|c|}{ Road } & & & \\
\hline $\begin{array}{l}\text { Baoshan South Road and Youza } \\
\text { Street }\end{array}$ & $\begin{array}{l}\text { Youza Street \& Qingnian } \\
\text { Road }\end{array}$ & $95 \%$ & {$[35 s, 310 s]$} & {$[0 s, 165 s]$} \\
\hline
\end{tabular}

\section{Conclusion}

Based on large-scale data, this paper constructs a network topology of city-level video detection equipment. For each pair of detection cross-section with upstream and downstream relationship in the topology table, the method of confidence self-adapting and truncation time self-adapting is adopted, and the method of steepest descent is used to construct the travel confidence time of road section. The validation results show that the travel confidence time can better describe the traffic status and travel time reliability of the road section. Compared with other recognition models, the recognition model constructed in this paper has three obvious advantages. First, the recognition model is based on real data completely, and there is no key assumption that does not conform to the actual conditions. For example, travel time conforms to normal distribution, Weill distribution, exponential distribution, etc. Second, it does not take the empirical value as the confidence threshold, but adopts an adaptive way, more in line with the actual situation of each section. Third, according to the actual situation of the data, it considers the lower limit of the interval of the reliable travel time, so that the travel confidence time. It is more accurate to describe the traveler's arrival probability within a given time. The interval of travel confidence time larger, the higher probability illegal parking occur. Road travel confidence time can be used as an important basis for data quality detection equipment, but also an important feature of road similarity. Accurate road travel confidence time is also the basis and key to build the entire urban traffic analysis platform, and even can do road network situation prediction. The results can be applied in other cities.

In this paper, the upper limit of travel confidence time is calculated based on the peak hours. The purpose is to get the maximum time for vehicles to pass through the section normally in the most congested period. However, it is not considered that the maximum congestion does not occur at the high level in some sections due to unreasonable signal timing scheme, more illegal parking or occupied traffic. During the peak period, the next research work of the author will focus on this aspect.

\section{Acknowledgment}

Foundation: Guizhou university, Guizhou Provincial Key Laboratory of Public Big Data Guizhou Guiyang,China (2017BDKFJJ012)

\section{References}

1. Lu Huapu L, Zhiyuan S. Big data and its applications in urban intelligent transportation system [J]. Journal of Transportation Systems Engineering and Information Technology, 15,5(2015).

2. Fengjie, F, Yue G, Dianhai W. Data quality analysis of link travel time based on HD smart gate [J]. Journal of ZheJiang University (Engineering Science), 50,9(2016).

3. Fei X, Zhang Y, Liu K, et al. Bayesian dynamic linear model with switching for real-time short-term freeway travel time prediction with license plate recognition data $[\mathrm{J}]$. Journal of Transportation Engineering, 139,11(2013).

4. Doughcrty M S, Kirby H R, EBOYLE R. The use of Neural Networks to recongnize and predict traffic congestion [J]. Traffic Engineering and Control, 34,6(1993).

5. Kazagli E, Koutsopoulos H. Estimation of arterial travel time from automatic number plate recognition data [J]. Transportation Research Record: Journal of the Transportation Research Board, 2391(2013).

6. Xiaoguang Y, Runlin C, Bing Z. Based on the data collected by the license auto-recognition system for predicting travel time $[\mathrm{J}]$. Journal of Transport Information and Safety, 3,23(2005).

7. Junting Z, Jing Z. Travel time estimation model based on spatial Markov chains [J]. Systems Engineering, 33,12 (2015).

8. Ang L, Shuo L, Lin L. Research on the calculation models of vehicle travel time on urban road segments [J]. Highway Engineering, 41,3(2016).

9. Lin W H, Kulkarni A, Mirchandani P. Short-term arterial travel time prediction for advanced traveler information systems [J]. Intelligent Transportation Systems. 8,3(2004).

10. Liu H X, et al. Real-time estimation of arterial travel time under congested conditions [J]. Transportmetrica,8,2(2012).

11. Daganzo C F. Urban gridlock: Macroscopic modeling and mitigation approaches[J]. Transportation Research Part B, 41,3(2007).

12. Lo H K, Luo XW, Siu B W Y. Degradable transport network: Travel time budget of travelers with heterogeneous risk aversion $[\mathrm{J}]$. Transportation Research Part B: Methodological, 40,9(2006).

13. Yong Z, Yu B, Xiaoguang Y. Travel time reliability in urban arterial networks [J]. Systems EngineeringTheory and Practice, 29,8(2009).

14. Linming Y, Ri-hui S. Path choosing algorithm based on travel time under stochastic time-varying characteristics $[\mathrm{J}]$. Journal of Transportation Systems Engineering and Information Technology, 17,5(2017).

15. Guiyan J, Ande C, Shifeng N. Traffic congestion identification method based on license plate 
recognition data [J].Journal of Haerbin institute of technology, 43,4(2011).

16. Fan Y, Xiaoguang Y. Urban road interrupted flow travel time bimodal distribution $[\mathrm{J}]$. Journal of TongJi University:Natural Science, 42,2(2014). 